\section{Continuous wear silicone hydrogel contact lenses and microbial keratitis}

MAN Whiting ${ }^{1}$, MK Raynor ${ }^{1}$, PB Morgan²,

P Galloway ${ }^{3}$, DM Tole ${ }^{3}$ and A Tullo ${ }^{1}$

\begin{abstract}
Like other lens types, the new generation of silicone hydrogel contact lenses can be associated with a spectrum of ocular complications. Most tend to be very minor, but serious and sight-threatening complications can occur. We present four such cases with microbial keratitis following extended wear of these lenses. Cultures were positive for Pseudomonas aeruginosa in three cases and all three of these suffered lasting visual impairment. We describe our findings and discuss possible risk factors.

Eye (2004) 18, 935-937. doi:10.1038/sj.eye.6701364

Published online 16 April 2004
\end{abstract}

Keywords: extended-wear; contact lens; microbial keratitis

Conventional hydrogel lenses worn overnight were associated with an unacceptably high incidence of microbial keratitis (MK). ${ }^{1-3}$

Consequently, extended wear of these lenses has been abandoned in many countries with notable exceptions being the United States and France. Low oxygen transmissibility ${ }^{4,5}$ resulting in low closed eye partial pressure of oxygen at the cornea, was thought to be the main factor responsible. Silicone hydrogel $(\mathrm{SiH})$ lenses have a much higher oxygen permeability (Dk) and have been approved in Europe, the United States and Australia for overnight wear. They are becoming increasingly popular due to the convenience of 30-day extended wear. However, their safety with respect to their incidence of $\mathrm{MK}$ is yet to be determined. We report four cases of $\mathrm{MK}$ presenting to two centres in the UK, three of which have resulted in lasting visual impairment.

\section{Case reports}

Case one

A 21-year-old male returned to the UK from a beach holiday. He presented to casualty at
Manchester Royal Eye Hospital (MREH) with a painful right eye for 1 day, with hand movements acuity and a $7 \mathrm{~mm} \times 8 \mathrm{~mm}$ infiltrated corneal ulcer with hypopyon (Photograph 1, top). He had been wearing a pair of $\mathrm{SiH}$ lenses for 1 week and had swum in the sea without goggles each day. Corneal scrape cultured moderate growth of Pseudomonas aeruginosa, sensitive to ofloxacin, ciprofloxacin, ceftazidime, and gentamicin. He was initially treated with intensive topical ofloxacin $0.3 \%$, with topical prednisolone added once healing was underway. After 2 months, acuity was 6/12 with pinhole with a $4.5 \mathrm{~mm}$ central scar and small epithelial defect.

\section{Case two}

A 28-year-old male presented to MREH with a painful red eye with a central infected ulcer after wearing an $\mathrm{SiH}$ lens overnight for 1 night only. He had previously worn hydrogel soft contact lenses on a daily wear basis for 7 years with no problems. At 3 days before

presentation, he commenced wearing a pair of $\mathrm{SiH}$ lenses for the first time. As instructed, he wore them during the day only for the first 2 days, removing them in the evening and disinfecting them with the solution provided by his contact lens practitioner. On the third day, he was to wear them overnight and be reviewed by the contact lens practitioner the following day. That day he woke with an extremely painful red left eye, unable to see, removed the lenses and presented to casualty at MREH. The acuity was 6/5 RE, light perception LE, with a dense central circular infiltrate and ulcer (Photograph 1 , middle). Both corneal scrape and contact lens cultures isolated moderate growths of $P$. aeruginosa sensitive to ofloxacin, ciprofloxacin, gentamicin, and imipenem. He responded to intensive guttae ofloxacin, with topical steroid added once improvement was apparent.

\author{
${ }^{1}$ Manchester Royal Eye \\ Hospital, Oxford Road \\ Manchester, M13 9WH, UK \\ ${ }^{2}$ Eurolens Research UMIST \\ Manchester, UK \\ ${ }^{3}$ Bristol Eye Hospital \\ Bristol, UK \\ Correspondence: M Raynor \\ Tel: + 4401612765522 \\ Fax: + 4401612726618 \\ E-mail: raymat@ \\ doctors.org.uk
}

Received: 30 July 2003 Accepted: 23 October 2003 Published online: 16 April 2004 


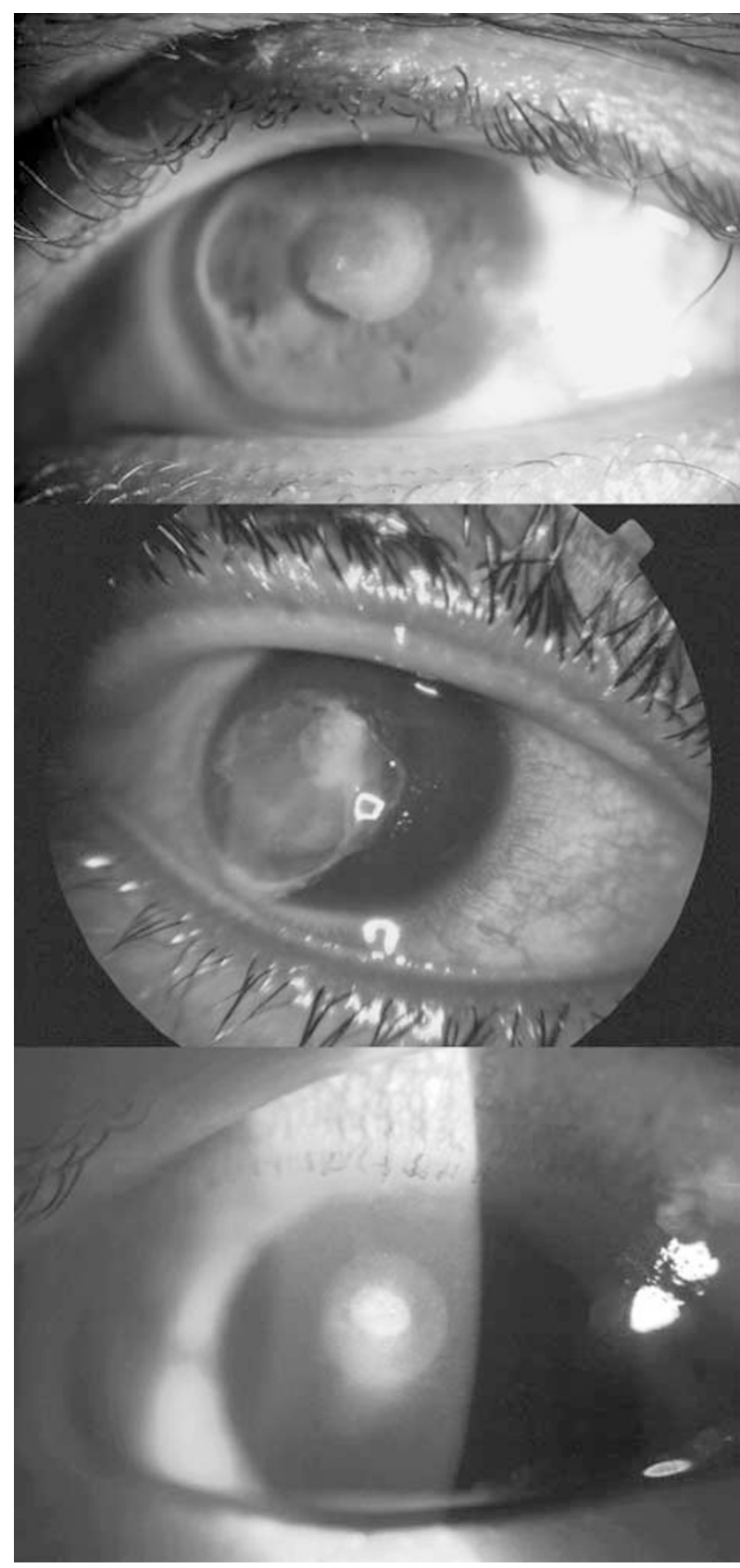

Photograph 1 (Top) case one - day 2, (middle) case two day 7 , (bottom) case three - day 7 .

After 3 months, with a depressed facetted dense paracentral corneal scar of $3.5 \mathrm{~mm}$ diameter, the acuity is 6/18 unaided not improving with pinhole.

\section{Case three}

A 42-year-old $\mathrm{SiH}$ contact lens wearer presented to Bristol Eye Hospital after developing some discomfort in the 3 eye three days previously and having removed the contact lens at that time. He had been wearing the lenses for 5 days, which his contact lens practitioner changed on a weekly basis. Visual acuity was reduced to $6 / 12$. A circular $2 \mathrm{~mm}$ corneal abscess was present in the upper one-third of the cornea (Photograph 1, bottom). Cultures yielded a scanty growth of coagulase-negative staphylococci. He responded well to cefuroxime and gentamicin over a period of 7 days. Topical steroids were administered for several days in order to reduce inflammation and scarring. He was discharged 6 weeks after his initial visit, with acuity returning to normal.

\section{Case four}

A 25-year-old male presented to MREH casualty on return from a holiday abroad. He had a central corneal ulcer in the right eye with $1 \mathrm{~mm}$ hypopyon. His vision was reduced to hand movements compared to $6 / 4$ in the left eye. He had been a user of monthly disposable $\mathrm{SiH}$ extended wear lenses for 2 years, but admitted that he sometimes took the lenses out at night and stored them in hydrogen peroxide and also that he had been swimming in the sea wearing lenses prior to developing his current problem.

A growth of $P$. aeruginosa was cultured from the corneal scrape and this was sensitive to ofloxacin. He responded to treatment with intensive topical ofloxacin $0.3 \%$ and later topical prednisolone but has been left with a $1.6 \mathrm{~mm}$ central facetted scar and reduced vision of $6 / 9$ at 1 month.

\section{Comment}

Microbial keratitis is potentially the most sightthreatening complication of contact lens wear, although improvements in lens materials, fitting and coatings, as well as better lens disinfectant solutions have all reduced the incidence of adverse events. ${ }^{6}$

The dangers of overnight wear were demonstrated when hydrogel lenses were widely worn for extended wear in the early 1990s and the incidence of microbial keratitis was as high as 13.3-20.9 per 10000 wearers per year for extended wear compared with 2.2-4.1 per 10000 for daily wear and two per 10000 for rigid lenses. ${ }^{1}$ These figures are similar to those of a large study of contactlens-associated MK in the Netherlands performed in the 1990s. ${ }^{3}$ The authors identified Pseudomonas and Serratia as the most common pathogens for MK overall and that Pseudomonas was associated with the poorest outcome in visual acuity. Even though lens materials have changed, the morbidity associated with Pseudomonas keratitis remains. This is shown by the fact that three of our four cases had scrapes that were cultured positive for Pseudomonas and all three suffered loss of best-corrected visual acuity (BCVA). 
The most recent advance in contact lens material has been the manufacture of an $\mathrm{SiH}$ polymer allowing a much higher $\mathrm{Dk} / \mathrm{t}$ contact lens. This increased $\mathrm{Dk} / \mathrm{t}$ is hoped to reduce the incidence of $\mathrm{MK}$ by better maintaining the integrity of the corneal epithelium, thus reducing the opportunity for bacterial penetration. These lenses are now being advocated for overnight wear and marketed as monthly extended wear contact lenses, (EWCLs). Another advantage of monthly extended wear is that there is no handling of the lenses for a month after CL insertion, minimising the chances of contaminating the lens.

The first prospective randomised control trial of these lenses had no cases of microbial keratitis in 504 patients wearing EWCLs for 7 or 30 days. $^{7}$ The first cases of MK in $\mathrm{SiH}$ EWCLs were reported in $2002 .^{8}$ The four cases were all male, between 16 and 22 years old and three had recently been swimming while wearing lenses.

Comparisons can be made with our cases, all males, two of whom had been swimming in the sea wearing lenses immediately prior to the development of $\mathrm{MK}$.

As of February 2003, 23 cases of MK with EWCLs have been reported worldwide, but the true incidence is not yet known. Holden et al. ${ }^{9}$ summarise data from a subgroup of these and propose that the risk of MK with $\mathrm{SiH}$ lenses is lower than that with conventional lenses when worn for extended wear. This may yet prove to be the case but prospective studies are required to establish the true incidence of $\mathrm{MK}$ in $\mathrm{SiH}$ lens wear.

\section{References}

1 Liesegang TJ. Contact lens-related microbial keratitis: Part I: epidemiology. Cornea 1997; 16: 125-131.

2 Stapleton F, Dart JKG, Minassian D. Risk factors with contact lens related suppurative keratitis. CLAO J 1993; 19: 204-210.

3 Cheng KH, Leung SL, Hoekman HW, Beekhuis WH, Mulder PG, Geerards AJ et al. Incidence of contact-lens-associated microbial keratitis and its related morbidity. Lancet 1999; 354: 181-185.

4 Dart JKG. Extended wear contact lenses, microbial keratitis, and the public health. Lancet 1991; 354: 174-175.

5 Fatt I. Comparative study of some physiologically important properties of six brands of disposable hydrogel contact lenses. CLAO J 1997; 23: 49-54.

6 Tomlinson A. Complications of Contact Lens Wear, CU. Mosby; St Louis, 1992

7 Nilsson SE. Seven-day extended wear and 30-day continuous wear of high oxygen transmissibility soft silicone hydrogel contact lenses: a randomized 1-year study of 504 patients. CLAO J 2001; 3: 125-136.

8 Lim L, Loughnan MS, Sullivan LJ. Microbial keratitis associated with extended wear of silicone hydrogel contact lenses. Br J Ophthalmol 2002; 86: 355-357.

9 Holden BA, Sweeney DF, Sankaridurg PR, Carnt N, Edwards $\mathrm{K}$, Stretton $\mathrm{S}$ et al. Microbial keratitis and vision loss with contact lenses. Eye Contact Lens 2003; 29 (1 Suppl): S131-S134. 